\title{
Present status of particle acceleration in relativistic outflows
}

\section{Benoît Cerutti*}

Univ. Grenoble Alpes, CNRS, IPAG, 38000 Grenoble, France

E-mail: benoit.cerutti@univ-grenoble-alpes.fr

\begin{abstract}
Relativistic outflows are ubiquitous in high-energy cosmic phenomena. Whether their reservoir of free energy at launch is in the form of bulk kinetic, magnetic or gravitational, it is ultimately channeled into energetic particles and non-thermal radiation. A key question is to understand how this transfer operates efficiently and under which conditions. Here, I review some of the recent developments in the modeling of two particle acceleration processes, namely relativistic diffusive shock acceleration and relativistic magnetic reconnection from the perspective of abinitio particle-in-cell simulations. These results and their astrophysical implications are briefly discussed in the context of relativistic outflows.
\end{abstract}

High Energy Phenomena in Relativistic Outflows VII - HEPRO VII

9-12 July 2019

Facultat de Física, Universitat de Barcelona, Spain

\footnotetext{
* Speaker.
} 


\section{Introduction}

The wide range of astrophysical sources exhibiting non-thermal radiation is proof that efficient particle acceleration is taking place and is ubiquitous in the Universe. The most efficient sources of high-energy particles are almost always associated with the activity of a neutron star or a black hole. In the vast majority of cases, the main energy reservoir can be gravitational, rotation or magnetic in nature ${ }^{1}$. The compact object then transforms this raw energy into a relativistic outflow either in the form of a wind as in pulsars [1], or in the form of a magnetized jet [2,3]. As the flow propagates away from the star, dissipation occurs which transforms the bulk kinetic energy and/or magnetic energy of the flow in the form of energetic particles which then radiate away their energy in the form of non-thermal radiation.

A key unknown in this chain of events is the nature of the dissipation process and how it operates to transform a macroscopic organized relativistic flow into non-thermal particle acceleration which occurs at microscopic scales. Two dissipative processes are most promising in explaining the observations and are currently drawing most of the community's attention: (i) relativistic shocks, and (ii) relativistic magnetic reconnection ${ }^{2}$. Here, I briefly review the recent developments in the modeling of particle acceleration in relativistic shocks (Sect. 2) and relativistic magnetic reconnection (Sect. 3) from the perspective of ab-initio particle-in-cell (PIC) simulations which have driven most of the progress in recent years. For simplicity, I will consider in the following pure electronpositron plasmas which is astrophysically adequate to describe pulsar winds, and more generally plasmas where all species are ultra-relativistic so that the rest mass ratio does not intervene in the dynamics (except radiative cooling which we will ignore here as well).

\section{Relativistic shocks}

Relativistic shocks convert the bulk kinetic energy of the outflow into non-thermal particle kinetic energy, as found in e.g., gamma-ray bursts afterglows, pulsar wind nebula termination shocks, relativistic jets (internal, recollimation, or external shocks). The plasma must also be collisionless to ensure that Coulomb collisions do not have time to thermalize the particles. This condition is almost always fulfilled in the context of relativistic jets and winds which are highly under-dense media. The theory of particle acceleration at collisionless shocks relies on the diffusive shock acceleration mechanism. Inspired from the first-order Fermi acceleration mechanism, this theory was formalized at the end of the seventies by [6, 7, 8]. In this framework, particles gain energy as they move back and forth across the shock front in a diffusive manner. In the limit of strong shocks, the theory predicts broad, steep $(\lesssim-2)$ power laws in the electron spectrum (see e.g., [9] for a recent review). The key ingredient for this mechanism to work is the presence of strong plasma turbulence at the kinetic scales, i.e., at the plasma skindepth and particle Larmor radius scale if the plasma is magnetized, both upstream and downstream the shock front in order to scatter particles on both sides. What is the nature of this turbulence, and can it sustain particle acceleration?

\footnotetext{
${ }^{1}$ One may argue that ultimately this was all gravitational energy in the first place.

${ }^{2}$ Other mechanisms under close scrutiny by the community in the context of relativistic outflows include relativistic turbulence [4] and shear-flow particle acceleration [5], but these two will not be reviewed here.
} 
PIC simulations came in handy in the last decade to address some of these questions from first principles.

\subsection{Unmagnetized shocks}

We first consider the simpler case of an unmagnetized shock, $\mathbf{B}=\mathbf{0}$. The numerical experiment often begins with a cold, relativistic beam of plasma heading toward a reflecting boundary for both the particles and the fields (perfectly conducting condition, e.g. [10], see however, [11]). To limit spurious heating of the beam by numerical Cherenkov radiation [12], the bulk Lorentz factor $\Gamma$ cannot be chosen arbitrarily large. It is often of the order $\Gamma \sim 10$. In addition, the beam has a small but finite co-moving temperature $\left(k T^{\prime} / m_{\mathrm{e}} c^{2} \ll 1 \sim 10^{-4}-10^{-2}\right)$. This setup captures the formation of a single shock only ${ }^{3}$.

The formation of the shock schematically proceeds along the following three main phases (Figure 1). In the first stage, the reflected flow heads backward and perfectly overlaps with the incoming flow (it is collisionless), such that the total plasma density jumps by a factor 2. The counter-streaming beam quickly becomes unstable to the Weibel instability [13,14], an electromagnetic instability growing over the (relativistic) plasma frequency timescale. The Weibel instability results in a series of self-generated magnetic filaments of alternating polarities on the skindepth scale, and seeds plasma turbulence. In the non-linear phase, plasma turbulence is mature enough to scatter and randomize particles efficiently in the downstream leading to the shock formation and to the expected Rankine-Hugoniot MHD jump conditions with a density contrast between the upstream and downstream close to 3 in $3 \mathrm{D}^{4}$ [15]. The plasma becomes effectively collisional except that the scattering centers are magnetic irregularities at the plasma scales self-generated and sustained by the flow.

The important element for the following is the formation of a filamentary structure in the upstream region, called the "precursor", which is composed of particles swimming back upstream from the shock front. These particles inform the plasma further upstream of the presence of the shock which effectively lead to a transition region where the incoming beam gradually slows down before reaching the shock front. As we will see further down, the spatial extent of the precursor is a direct measure of the maximum energy that the particles can reach. After a long integration time $\left(t \gtrsim 1000 \omega_{\mathrm{pe}}^{-1}\right.$ plasma frequency time), a high-energy power-law component with a -2.4 index emerges from the thermalized background followed by an exponential cutoff [10]. The maximum energy of the distribution increases as $\propto \sqrt{t}[16,17]$, confirming the diffusive nature of the acceleration process, but without any sign of saturation (left panel in Figure 2). The study of individual particle trajectories reveals that particles gain energy as they scatter back and forth across the shock as predicted by the diffusive shock acceleration mechanism.

\subsection{Magnetized shocks and maximum particle energy}

The efficiency of diffusive shock acceleration depends sensitively on the background magnetic

\footnotetext{
${ }^{3}$ Reverse or forward shock depending on the definition of the upstream medium, the simulation frame being in the contact discontinuity frame (here, the simulation wall).

${ }^{4}$ This holds as long as it is $3 \mathrm{D}$ in the velocity space.
} 
$\mathbf{y}$

\ Phase 1: The two flows overlap without interacting
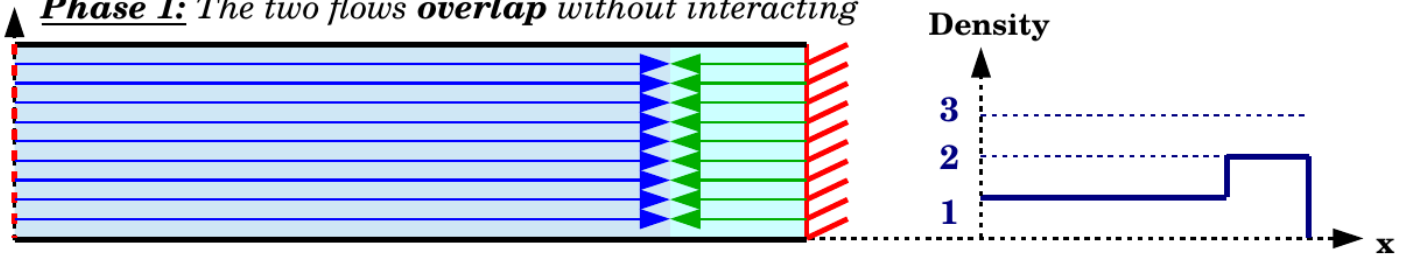

Phase 2: Electromagnetic counter-streaming instabilities grows (linear phase)

$\mathbf{y}$
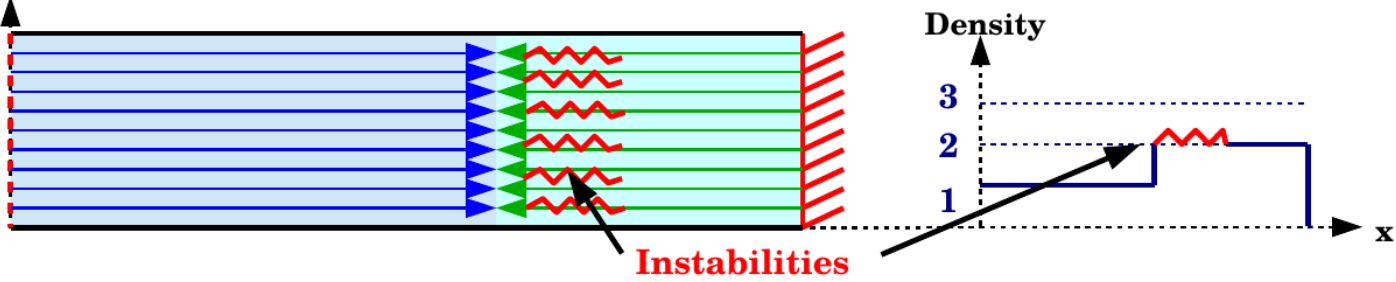

Phase 3: Non-linear phase, the shock form and particle acceleration begins

$\mathbf{y}$

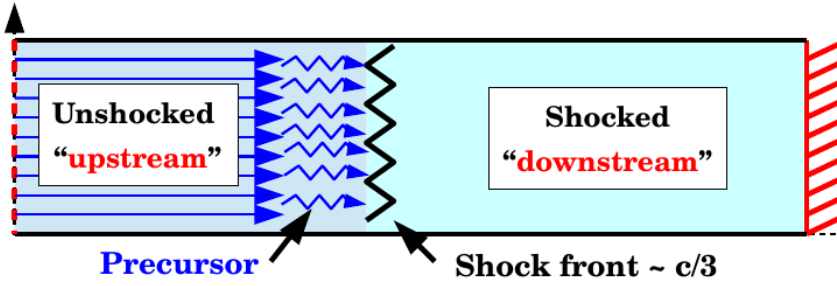

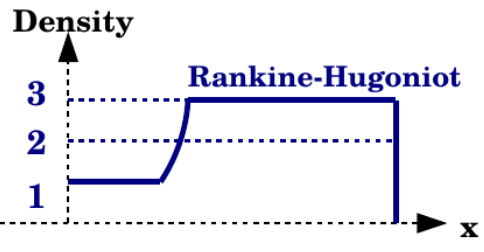

Figure 1: Schematic stages of unmagnetized relativistic shock formation in PIC simulations.
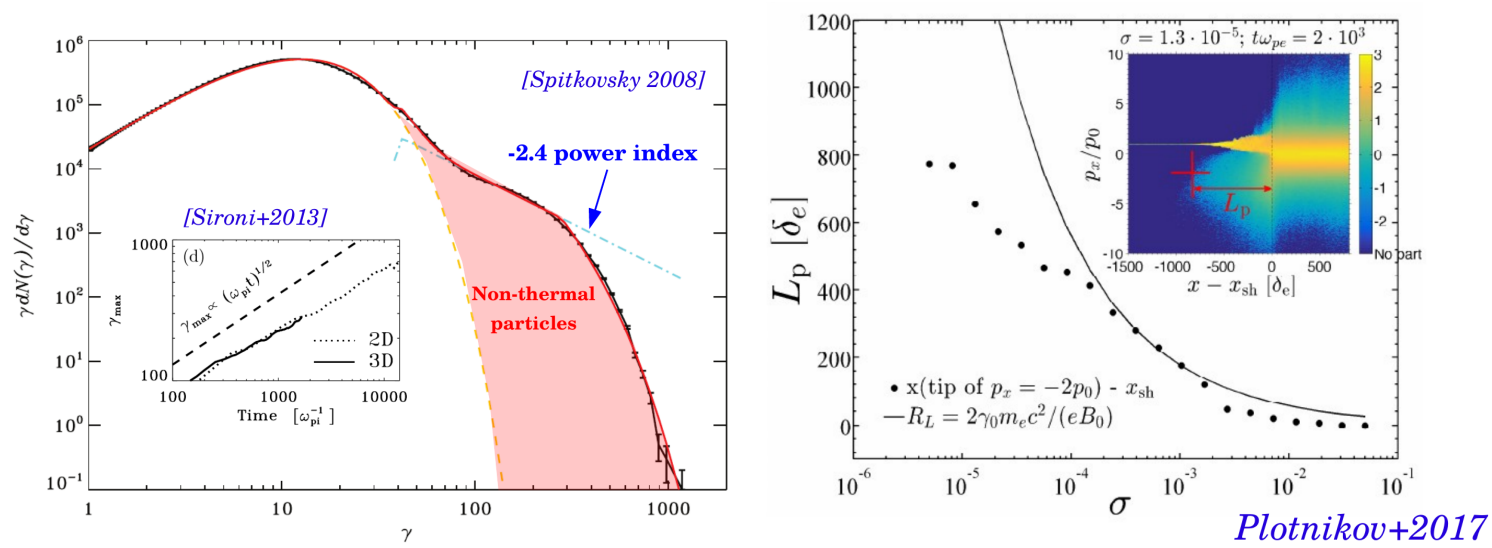

Figure 2: Left: Particle energy spectrum obtained by Spitkovsky [10] in the limit of an unmagnetized relativistic $(\Gamma=15)$ shock. A clear sign of non-thermal particle acceleration in the form of a -2.4 power law is visible after an long integration time $\left(t \gtrsim 1000 \omega_{\mathrm{pe}}^{-1}\right)$. Inset: The high-energy cutoff grows as $\gamma_{\max } \propto \sqrt{t}$ without any sign of saturation. Figure adapted from [10] and [16]. Right: Length of the precursor as a function of the plasma magnetization as measured in PIC simulations and compared with the Larmor radius of the returning particles upstream. Figure adapted from [17]. 
field strength. More specifically, it depends on the plasma magnetization parameter defined as

$$
\sigma=\frac{B_{0}^{2}}{4 \pi \Gamma n_{0} m_{\mathrm{e}} c^{2}}
$$

where $n_{0}$ and $B_{0}$ are, respectively, the background plasma density and background magnetic field. Ultra-relativistic magnetized shocks are necessarily quasi-perpendicular ${ }^{5}$ in the downstream frame due to Lorentz contraction and field compression. The numerical configuration is identical to the one described above with the addition of a uniform magnetic field and the ideal motion electric field $\mathbf{E}=-\mathbf{V} \times \mathbf{B} / c$.

At low but finite magnetization $\left(\sigma \lesssim 10^{-2}\right)$, the solution closely resembles the unmagnetized case $(\sigma=0)$ with one important difference: the length of the precursor developing upstream shrinks with increasing magnetization. Analytical arguments predicted that it should scale as the Larmor radius of the returning particles and therefore be infinitely large at $\sigma=0$ down to a finite value ([18], right panel in Figure 2). This result has been confirmed later on by PIC simulations [16, 17]. Beyond $\sigma \gtrsim 10^{-2}$, the particle Larmor radius becomes too small to allow the particles to make significant excursions in the upstream before being advected downstream (magnetic reflection). Therefore, no particles can return in the upstream, the precursor disappears and particle acceleration is quenched.

The maximum particle energy that can possibly be reached at a relativistic shock depends on the length of the precursor. Sironi et al. (2013) [16] reported clear signs of the saturation of highenergy cutoff energy as $\gamma_{\max } / \Gamma \propto \sigma^{-1 / 4}$. They further argue that while this scaling is particularly relevant for explaining particle acceleration in gamma-ray burst afterglows, it is most likely not so favourable in pulsar wind nebulae.

\subsection{The puzzling case of the Crab pulsar wind nebula}

Pulsars blow ultra-relativistic $\left(\Gamma \sim 10^{2}-10^{6}\right)$, magnetized cold winds which terminate in the form of a nebula where particles are randomized. The magnetization inside the nebula is rather uncertain, but recent global 3D MHD simulations of the Crab pulsar wind nebulae suggest that it could be of order $\sigma \sim$ a few and may be even higher [19], in any case much higher than $\sigma \sim 10^{-2}$. Yet, observations show a clear power-law spectrum spanning over 5-6 orders of magnitude from $\mathrm{X}$-rays to gamma rays in the synchrotron spectrum meaning that an electron distribution over 3 decades with a power-law index close to the first-order Fermi acceleration prediction of -2.2 is present in the nebula [20]. While most of the other features of the nebula are now rather well understood, particle acceleration in pulsar wind nebulae remains a complete mystery [21, 22]. Several other mechanisms have been proposed in the literature (e.g., cyclotron wave acceleration $[23,24]$, driven reconnection [25, 26]), but none of them seems to perfectly do the trick. More recent models have been advocating the effect of externally driven turbulence which could maintain the Fermi mechanism at work, for instance induced by corrugations in the shock front [27, 28], or by the global, large-scale dynamics of the nebula [19, 29]. Another recent model considers the equatorial plane where, by construction, the magnetic field vanishes and therefore diffusive shock acceleration can operate [30]. The key of this mystery may be found in the strong latitudinal

\footnotetext{
${ }^{5}$ The direction of the magnetic field is perpendicular to the shock normal
} 
dependence of the pulsar shock front properties (in particular the magnetic field strength). Current PIC studies have solely focused on uniform configurations, without any dependence on the plasma properties along the shock front which is not realistic on large scales in pulsar wind nebulae. It would be interesting to investigate such a case in the future.

\section{Relativistic magnetic reconnection}

As we will see further in this section, in contrast to relativistic shocks, relativistic magnetic reconnection is most efficient at accelerating particles at high plasma magnetization therefore making these two processes complementary. Magnetic reconnection is by definition a change in the magnetic field line topology. This rearrangement leads to magnetic dissipation via the formation of current sheets, plasma heating and particle acceleration. Reconnection proceeds in the relativistic regime when the Alfvén speed approaches the speed of light [31, 32, 33], i.e.,

$$
V_{\mathrm{A}}=c \sqrt{\frac{\sigma}{\sigma+1}} \approx c, \text { for } \sigma \gtrsim 1
$$

with

$$
\sigma=\frac{B_{0}^{2}}{4 \pi n_{0} m_{\mathrm{e}} c^{2}}
$$

where $B_{0}$ is the reconnecting component of the magnetic field, and $n_{0}$ the background plasma density. Another way to convince ourselves that reconnection proceeds in the relativistic regime is to look at the definition of $\sigma$ itself. It represents how much more time there is energy density in the field (the reservoir) in comparison with the plasma rest mass energy density. Therefore, if a significant fraction of $B_{0}^{2}$ is dissipated and channelled to particles, the plasma necessarily becomes relativistic for $\sigma \gtrsim 1$. This regime is achieved in very peculiar astrophysical environments, most certainly in pulsar magnetospheres [34], pulsar winds [21] and black hole magnetospheres [2], most likely in relativistic jets [35, 36], black hole coronae [37, 38], pulsar wind nebulae [39], and possibly gamma-ray bursts [40].

Some of the key questions in the field are how fast does reconnection proceed (what is the reconnection rate) and how is it mediated/triggered? How efficient is reconnection at accelerating particles and where does it take place in nature? Significant progress on these issues have been made recently, here again mostly thanks to the use of PIC simulations. I highlight some of these results below, focusing on particle acceleration. For recent and more detailed reviews on the field, see $[41,42,43]$.

\subsection{Reconnection dynamics}

Most numerical studies begin with a relativistic Harris current sheet configuration [44] (see however, e.g., [45] for an initially force-free configuration), a steady-state kinetic plasma equilibrium. The magnetic field reverses its polarity over a distance $\delta$ as $B=B_{0} \tanh (y / \delta)$ where an electric current flows such that $\mathbf{J}=(4 \pi / c) \nabla \times \mathbf{B}$ is carried by the plasma. The system is in pressure balance between the magnetic pressure and the plasma thermal pressure in the sheet. The initial layer thickness is of order the plasma skindepth. For a long and thin current sheet, this equilibrium 
quickly becomes unstable to the relativistic tearing mode $[46]^{6}$. The current sheet fragments in a series of alternating magnetic $\mathrm{O}$ - and X-points, corresponding to plasma-filled islands or referred later as "plasmoids" separated by secondary current sheets. Secondary current sheets as they form can in turn become unstable and form more islands (the plasmoid instability, [51]). Islands can also merge to form bigger ones so that in the non-linear stage the system is composed of a distribution of magnetic islands sizes (see Figure 3). As plasma is advected inside magnetic islands by the release of magnetic tension at X-points, there is an inflow of fresh plasma from the background medium. The process proceeds as long as the sheet is fed by fresh reconnecting magnetic flux coming from upstream on either side of the sheet. The ratio of the incoming plasma velocity at X-points and the outgoing plasma velocity accelerated by the magnetic tension away from the $\mathrm{X}$-points towards the islands gives a fair estimate of the dimensionless reconnection rate, $\beta_{\text {rec }}=V_{\text {in }} / V_{\text {out }}$. Today, all studies seem to agree that relativistic reconnection is very fast, with $\beta_{\text {rec }} \sim 0.1-0.3$, regardless of the plasma magnetization as long as $\sigma \gtrsim 1$.

\subsection{Particle acceleration and maximum energy}

There is also a consensus today that relativistic reconnection is efficient at dissipating magnetic energy and at accelerating particles. Different groups over the last two decades came to the same conclusion that reconnection naturally produces hard particle spectra at high magnetization (e.g., [49, 52, 45], Figure 4). The spectrum hardens with increasing $\sigma$ and seems to saturate to nearly $\gamma^{-1.2}$. A physical origin of such power-law indices is still debated and poorly understood. But the simple fact that reconnection produces power laws harder than -2 already have strong implications on the maximum particle energy. Indeed, this means that the energy distribution $\gamma^{2} d N / d \gamma$ is peaking at $\gamma_{\max }$. Conservation of energy tells us that $\gamma_{\max }$ cannot be arbitrarily large and should depend on how much magnetic energy there is available per particle, i.e., $\sigma$. Werner et al. (2016) found that a good fit of the maximum particle cut-off energy for large box size is $\gamma_{\max } \approx 4 \sigma$ [52].

To gain more physical insights, it is usually instructive to look at individual particle trajectories. This study indicates that particles undergo multiple episodes of acceleration along their history [57]. The first episode is usually an impulsive acceleration by the reconnection electric field induced at X-points. This field is maintained by non-ideal terms in the generalized Ohm's law. In pair plasmas, the off-diagonal terms of the plasma pressure tensor plays the dominant role within the current layer [53]. The total energy gained by the particle depends on how close it has entered from the center of the X-point because it determines the lifetime of the particle in the accelerating region before it is pushed away towards plasmoids by the reconnected magnetic field [55, 49]. The energy gain is proportional to time, consistent with a linear-like acceleration. Particle follows the relativistic analog of Speiser orbits $[54,55]$ as they move back and forth across the current layer where the magnetic field reverses. The particle distribution is also highly anisotropic due to the preferential direction of the reconnection electric field, the most energetic particles being the most beamed [56]. Once they reached an island they are confined by the magnetic field, and their directions are randomized. Island mergers can lead to an extra particle energy boost of a similar nature

\footnotetext{
${ }^{6}$ In full 3D, the sheet is also kink unstable with a similar growth rate [47, 48]. There is a competition between these two modes in the early stages but the tearing instability seems to dominate the non-linear evolution of the system $[49,50]$.
} 


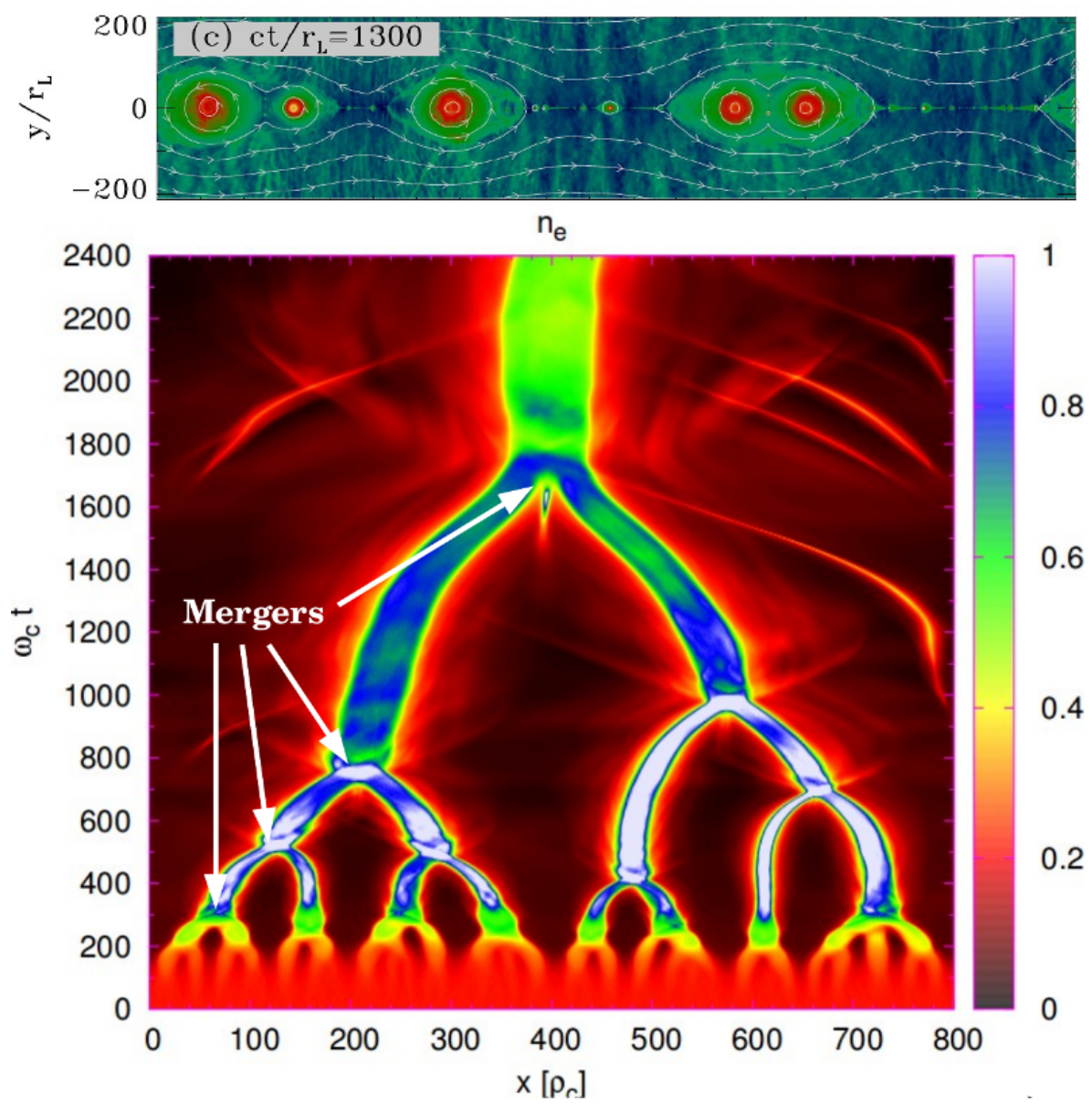

Figure 3: Top: Plasma density snapshot of a 2D PIC simulation in the non-linear phase of the plasmoid instability. Bottom: Space-time diagram showing the merging tree of plasmoids as the simulation evolves leading to bigger and bigger structure until there is just one left filling the simulation box. Figure adapted from [49] and [57].

$[49,57]$. In this case, a secondary current sheet forms perpendicular to the main one, at the interface between both islands. The main physical scenario of particle acceleration depicted here seems to hold in full 3D simulations even in the absence of a guide field component (i.e., perpendicular to the reconnection plane) [49, 50].

A recent study by Petropoulou \& Sironi (2018) [58] suggests that another regime of particle acceleration has been missed by previous studies at even larger system sizes. They found that while the early evolution of reconnection is consistent with linear-like acceleration episodes leading to $\gamma_{\max } \propto t$ and hard spectra, the late evolution is dominated by a slower, diffusive-like acceleration within large magnetic islands such that $\gamma_{\max } \propto \sqrt{t}$. This is accompanied by a spectral steepening allowing the maximum energy to grow significantly above the $4 \sigma$ limit without any clear sign of saturation. This extra acceleration is attributed to the slow compression of magnetic islands. It is a promising way to accelerate particles to very high energies in astrophysical environments where $\sigma$ may not be very high (of order unity), except of course in pulsar and black hole magnetospheres where $\sigma \gg 1$ is most likely guaranteed. If island compression is indeed the dominant acceleration 

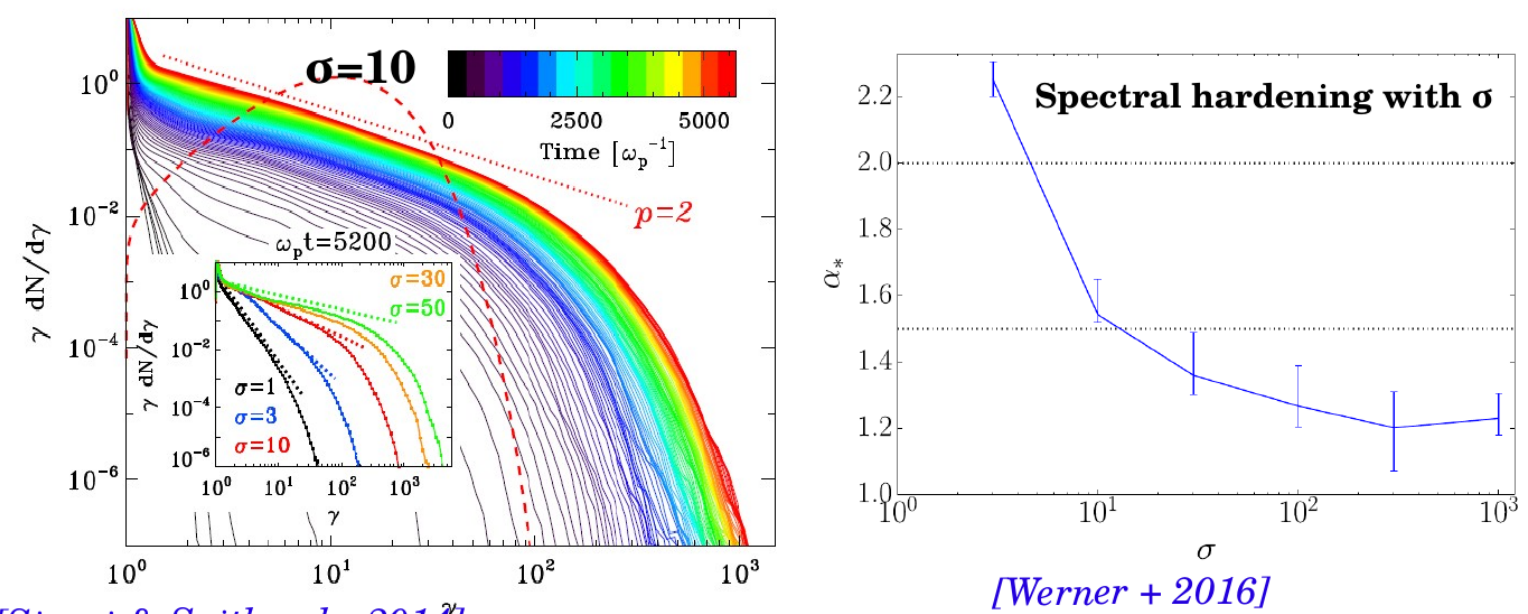

[Sironi \& Spitkovsky 2014]

Figure 4: Left: Time evolution of the particle energy spectrum in a 2D PIC relativistic reconnection simulation with $\sigma=10$ (other values are shown in the inset plot). Right: Power-law index of the particle spectrum as function of $\sigma$. Figure adapted from [49] and [52].

process in reconnection, this also implies that the role of the kinetic scale physics at X-points becomes secondary and it could be seen as an injection mechanism. Ironically, we can speculate that the full PIC simulation artillery may not be needed in the future, and resistive MHD simulations with test particles could come handy to explore larger, more realistic system sizes at a smaller numerical cost without missing much of the dominant particle acceleration physics.

\section{Summary}

(i) Relativistic shocks: The Weibel instability mediates the formation of the shock by selfgenerated magnetic turbulence. The degree of turbulence both upstream and downstream the shock to allow diffusive shock acceleration to operate is effective only at weakly magnetized shocks $\left(\sigma \lesssim 10^{-2}\right)$. At higher $\sigma$, magnetic reflection prevents the particles from being scattered at the shock front and no turbulence upstream can be sustained leading to no particle acceleration. When effective, particle acceleration is slow $(\propto \sqrt{t}$ ) and a steep power-law tail (of index $\lesssim-2$ ) forms whose maximum energy is limited by $\gamma_{\max } \sim \Gamma \sigma^{-1 / 4}$. Relativistic diffusive shock acceleration is a good candidate for explaining gamma-ray burst afterglows, but fails at explaining particle acceleration in pulsar wind nebulae. Global inhomogeneous model could be the key to this mystery.

(ii) Relativistic magnetic reconnection: Fast reconnection is mediated by the fragmentation of the current sheet into a series of multiple $\mathrm{X}$-points separated by magnetic islands. In highly magnetized plasmas $(\sigma \gtrsim 1)$, particle acceleration is fast ( $\propto t$ in the early phases) and efficient. Particles are accelerated during multiple impulsive acceleration episodes by the reconnection electric field either at the main X-points or at large plasmoid merger events. Relativistic reconnection produces hard $(\gtrsim-2)$ particle spectrum which are in return limited by $\sigma$. A slower acceleration mechanism within contracting islands at large timescales accompanied by a spectral steepening allows to go significantly beyond the $\sigma$ limit. Therefore, relativistic reconnection is a promising mechanism 
for accelerating particles in a wide range of astrophysical environments, from mildly magnetized environments like jets and pulsar wind nebulae to the ultra-magnetized environments of pulsar and black hole magnetospheres.

\section{Acknowledgments}

I would like to thank the organizers for their invitation and for their hospitality during the HEPRO-VII meeting. I would like also to thank the referee for valuable comments on the manuscript. This work was supported the Programme National des Hautes Énergie of CNRS/INSU and CNES.

\section{References}

[1] Michel, F. C. 1969. Relativistic Stellar-Wind Torques. The Astrophysical Journal 158, 727.

[2] Blandford, R. D., Znajek, R. L. 1977. Electromagnetic extraction of energy from Kerr black holes. Monthly Notices of the Royal Astronomical Society 179, 433.

[3] Blandford, R. D., Payne, D. G. 1982. Hydromagnetic flows from accretion disks and the production of radio jets.. Monthly Notices of the Royal Astronomical Society 199, 883.

[4] Zhdankin, V., Werner, G. R., Uzdensky, D. A., Begelman, M. C. 2017. Kinetic Turbulence in Relativistic Plasma: From Thermal Bath to Nonthermal Continuum. Physical Review Letters 118, 055103 .

[5] Rieger, F. M. 2019. An Introduction to Particle Acceleration in Shearing Flows. Galaxies 7, 78.

[6] Axford, W. I., Leer, E., Skadron, G. 1977. The Acceleration of Cosmic Rays by Shock Waves. International Cosmic Ray Conference 132.

[7] Blandford, R. D., Ostriker, J. P. 1978. Particle acceleration by astrophysical shocks.. The Astrophysical Journal 221, L29.

[8] Bell, A. R. 1978. The acceleration of cosmic rays in shock fronts - I.. Monthly Notices of the Royal Astronomical Society 182, 147.

[9] Pelletier, G., Bykov, A., Ellison, D., Lemoine, M. 2017. Towards Understanding the Physics of Collisionless Relativistic Shocks. Relativistic Collisionless Shocks. Space Science Reviews 207, 319.

[10] Spitkovsky, A. 2008. Particle Acceleration in Relativistic Collisionless Shocks: Fermi Process at Last?. The Astrophysical Journal 682, L5.

[11] Ardaneh, K., Cai, D., Nishikawa, K.-I., Lembége, B. 2015. Collisionless Weibel Shocks and Electron Acceleration in Gamma-Ray Bursts. The Astrophysical Journal 811, 57.

[12] Greenwood, A. D., Cartwright, K. L., Luginsland, J. W., Baca, E. A. 2004. On the elimination of numerical Cerenkov radiation in PIC simulations. Journal of Computational Physics 201, 665.

[13] Weibel, E. S. 1959. Spontaneously Growing Transverse Waves in a Plasma Due to an Anisotropic Velocity Distribution. Physical Review Letters 2, 83.

[14] Medvedev, M. V., Loeb, A. 1999. Generation of Magnetic Fields in the Relativistic Shock of Gamma-Ray Burst Sources. The Astrophysical Journal 526, 697.

[15] Kennel, C. F., Coroniti, F. V. 1984. Confinement of the Crab pulsar's wind by its supernova remnant.. The Astrophysical Journal 283, 694. 
[16] Sironi, L., Spitkovsky, A., Arons, J. 2013. The Maximum Energy of Accelerated Particles in Relativistic Collisionless Shocks. The Astrophysical Journal 771, 54.

[17] Plotnikov, I., Grassi, A., Grech, M. 2018. Perpendicular relativistic shocks in magnetized pair plasma. Monthly Notices of the Royal Astronomical Society 477, 5238.

[18] Lemoine, M., Pelletier, G. 2010. On electromagnetic instabilities at ultra-relativistic shock waves. Monthly Notices of the Royal Astronomical Society 402, 321.

[19] Porth, O., Komissarov, S. S., Keppens, R. 2014. Three-dimensional magnetohydrodynamic simulations of the Crab nebula. Monthly Notices of the Royal Astronomical Society 438, 278.

[20] Meyer, M., Horns, D., Zechlin, H.-S. 2010. The Crab Nebula as a standard candle in very high-energy astrophysics. Astronomy and Astrophysics 523, A2.

[21] Kirk, J. G., Lyubarsky, Y., Petri, J. 2009. The Theory of Pulsar Winds and Nebulae. Astrophysics and Space Science Library 421.

[22] Kargaltsev, O., Cerutti, B., Lyubarsky, Y., Striani, E. 2015. Pulsar-Wind Nebulae. Recent Progress in Observations and Theory. Space Science Reviews 191, 391.

[23] Hoshino, M., Arons, J., Gallant, Y. A., Langdon, A. B. 1992. Relativistic Magnetosonic Shock Waves in Synchrotron Sources: Shock Structure and Nonthermal Acceleration of Positrons. The Astrophysical Journal 390, 454.

[24] Amato, E., Arons, J. 2006. Heating and Nonthermal Particle Acceleration in Relativistic, Transverse Magnetosonic Shock Waves in Proton-Electron-Positron Plasmas. The Astrophysical Journal 653, 325 .

[25] Lyubarsky, Y. E. 2003. The termination shock in a striped pulsar wind. Monthly Notices of the Royal Astronomical Society 345, 153.

[26] Sironi, L., Spitkovsky, A. 2011. Acceleration of Particles at the Termination Shock of a Relativistic Striped Wind. The Astrophysical Journal 741, 39.

[27] Lemoine, M. 2016. A corrugated termination shock in pulsar wind nebulae?. Journal of Plasma Physics 82, 635820401.

[28] Demidem, C., Lemoine, M., Casse, F. 2018. Relativistic magnetohydrodynamical simulations of the resonant corrugation of a fast shock front. Monthly Notices of the Royal Astronomical Society 475, 2713.

[29] Olmi, B., Del Zanna, L., Amato, E., Bandiera, R., Bucciantini, N. 2014. On the magnetohydrodynamic modelling of the Crab nebula radio emission. Monthly Notices of the Royal Astronomical Society 438, 1518.

[30] Giacinti, G., Kirk, J. G. 2018. Acceleration of X-Ray Emitting Electrons in the Crab Nebula. The Astrophysical Journal 863, 18.

[31] Blackman, E. G., Field, G. B. 1994. Kinematics of relativistic magnetic reconnection. Physical Review Letters 72, 494.

[32] Lyutikov, M., Uzdensky, D. 2003. Dynamics of Relativistic Reconnection. The Astrophysical Journal $589,893$.

[33] Lyubarsky, Y. E. 2005. On the relativistic magnetic reconnection. Monthly Notices of the Royal Astronomical Society 358, 113. 
[34] Cerutti, B., Beloborodov, A. M. 2017. Electrodynamics of Pulsar Magnetospheres. Space Science Reviews 207, 111.

[35] Romanova, M. M., Lovelace, R. V. E. 1992. Magnetic field, reconnection and particle acceleration in extragalactic jets.. Astronomy and Astrophysics 262, 26.

[36] Giannios, D., Uzdensky, D. A., Begelman, M. C. 2009. Fast TeV variability in blazars: jets in a jet. Monthly Notices of the Royal Astronomical Society 395, L29.

[37] Galeev, A. A., Rosner, R., Vaiana, G. S. 1979. Structured coronae of accretion disks.. The Astrophysical Journal 229, 318.

[38] Goodman, J., Uzdensky, D. 2008. Reconnection in Marginally Collisionless Accretion Disk Coronae. The Astrophysical Journal 688, 555.

[39] Cerutti, B., Werner, G. R., Uzdensky, D. A., Begelman, M. C. 2014. Gamma-ray flares in the Crab Nebula: A case of relativistic reconnection?a). Physics of Plasmas 21, 056501.

[40] Drenkhahn, G., Spruit, H. C. 2002. Efficient acceleration and radiation in Poynting flux powered GRB outflows. Astronomy and Astrophysics 391, 1141.

[41] Zweibel, E. G., Yamada, M. 2009. Magnetic Reconnection in Astrophysical and Laboratory Plasmas. Annual Review of Astronomy and Astrophysics 47, 291.

[42] Hoshino, M., Lyubarsky, Y. 2012. Relativistic Reconnection and Particle Acceleration. Space Science Reviews 173, 521.

[43] Kagan, D., Sironi, L., Cerutti, B., Giannios, D. 2015. Relativistic Magnetic Reconnection in Pair Plasmas and Its Astrophysical Applications. Space Science Reviews 191, 545.

[44] Kirk, J. G., Skjæraasen, O. 2003. Dissipation in Poynting-Flux-dominated Flows: The $\sigma$-Problem of the Crab Pulsar Wind. The Astrophysical Journal 591, 366.

[45] Guo, F., Li, H., Daughton, W., Liu, Y.-H. 2014. Formation of Hard Power Laws in the Energetic Particle Spectra Resulting from Relativistic Magnetic Reconnection. Physical Review Letters 113, 155005.

[46] Zelenyi, L. M., Krasnoselskikh, V. V. 1979. Relativistic Modes of Tearing Instability in a Background Plasma. Soviet Astronomy 23, 460.

[47] Zenitani, S., Hoshino, M. 2007. Particle Acceleration and Magnetic Dissipation in Relativistic Current Sheet of Pair Plasmas. The Astrophysical Journal 670, 702.

[48] Cerutti, B., Werner, G. R., Uzdensky, D. A., Begelman, M. C. 2014. Three-dimensional Relativistic Pair Plasma Reconnection with Radiative Feedback in the Crab Nebula. The Astrophysical Journal 782, 104.

[49] Sironi, L., Spitkovsky, A. 2014. Relativistic Reconnection: An Efficient Source of Non-thermal Particles. The Astrophysical Journal 783, L21.

[50] Werner, G. R., Uzdensky, D. A. 2017. Nonthermal Particle Acceleration in 3D Relativistic Magnetic Reconnection in Pair Plasma. The Astrophysical Journal 843, L27.

[51] Uzdensky, D. A., Loureiro, N. F., Schekochihin, A. A. 2010. Fast Magnetic Reconnection in the Plasmoid-Dominated Regime. Physical Review Letters 105, 235002.

[52] Werner, G. R., Uzdensky, D. A., Cerutti, B., Nalewajko, K., Begelman, M. C. 2016. The Extent of Power-law Energy Spectra in Collisionless Relativistic Magnetic Reconnection in Pair Plasmas. The Astrophysical Journal 816, L8. 
[53] Bessho, N., Bhattacharjee, A. 2005. Collisionless Reconnection in an Electron-Positron Plasma. Physical Review Letters 95, 245001.

[54] Speiser, T. W. 1965. Particle Trajectories in Model Current Sheets, 1, Analytical Solutions. Journal of Geophysical Research 70, 4219.

[55] Cerutti, B., Uzdensky, D. A., Begelman, M. C. 2012. Extreme Particle Acceleration in Magnetic Reconnection Layers: Application to the Gamma-Ray Flares in the Crab Nebula. The Astrophysical Journal 746, 148.

[56] Cerutti, B., Werner, G. R., Uzdensky, D. A., Begelman, M. C. 2012. Beaming and Rapid Variability of High-energy Radiation from Relativistic Pair Plasma Reconnection. The Astrophysical Journal 754, L33.

[57] Nalewajko, K., Uzdensky, D. A., Cerutti, B., Werner, G. R., Begelman, M. C. 2015. On the Distribution of Particle Acceleration Sites in Plasmoid-dominated Relativistic Magnetic Reconnection. The Astrophysical Journal 815, 101.

[58] Petropoulou, M., Sironi, L. 2018. The steady growth of the high-energy spectral cut-off in relativistic magnetic reconnection. Monthly Notices of the Royal Astronomical Society 481, 5687. 